\title{
Gestión de gobierno abierto en las instituciones públicas: una revisión de la literatura
}

Management of open government in public institutions: a literature review

Gestão de governo aberto nas instituições públicas: uma revisão da literatura

\author{
Amelia Eunice Maldonado-Lozano \\ aemaldonadom@ucvvirtual.edu.pe \\ Universidad César Vallejo (UCV) - Perú \\ ORCID: 0000-0001-8137-1361
}

Luis Paredes-Aguilar

luparedasa@ucvvirtual.edu.pe

Universidad César Vallejo (UCV) - Perú

ORCID: 0000-0002-1375-179X

\author{
Gabriela del Pilar Palomino Alvarado \\ dpalominoal@ucvvirtual.edu.pe \\ Universidad César Vallejo (UCV) - Perú \\ ORCID: 0000-0003-0851-4375
}

\begin{abstract}
RESUMEN
La gestión de gobierno abierto constituye la clave para lograr la transparencia y confianza de la población en las instituciones públicas, que impide que la información generada sea utilizada en forma permanente y con seguridad; facilitando el conocimiento de las acciones que la gestión pública viene desarrollando. El objetivo es examinar e analizar la evidencia científica existente referente a la gestión de gobierno abierto en las instituciones públicas, recurriendo a la base de datos de EBSCO, SCOPUS y SCIENCE DIRECT en inglés y en español de los últimos cuatro años, utilizando el término "gestión de gobierno abierto", descartándose artículos que no sean arbitradas y académicas. Teniendo como resultados que para transparentar la gestión en las instituciones públicas debe desarrollarse nuevos paradigmas en la gestión del gobierno abierto, iniciando con acciones de mejora en la generación de la información para su aprovechamiento de la población, en diferentes acciones de índole social o económico, sin sesgar la información restringiéndola a uso propio de la institución pública. Se concluye señalando que para una gestión de gobierno abierto eficiente y eficaz, debemos tomar en cuenta los retos que significa transparentar la gestión con el uso de datos abiertos para uso y beneficio de la población, transparentando las acciones y recursos que la administración pública invierte. Palabras clave / Descriptores: Gobierno abierto, datos abiertos, apertura de los gobiernos públicos, gestión pública.
\end{abstract}

\begin{abstract}
The management of open government constitutes the key to achieve transparency and trust of the population in public institutions, which prevents the information generated from being used permanently and safely; facilitating knowledge of the actions that public management has been developing. The objective is to examine and analyze the existing scientific evidence regarding the management of open government in public institutions, using the EBSCO, SCOPUS and SCIENCE DIRECT databases in English and Spanish for the last four years, using the term "open government management", discarding articles that are not refereed and academic. Having as results that to make management transparent in public institutions, new paradigms must be developed in the management of open government, starting with actions to improve the generation of information for its use by the population, in different actions of a social or economic nature, without biasing the information, restricting it to the use of the public institution. It concludes by noting that for efficient and effective open government management, we must take into account the challenges of transparent management with the use of open data for the use and benefit of the population, making transparent the actions and resources that the public administration invests.
\end{abstract}

Keywords: Open government, open data, openness of public governments, public management.

\section{RESUMO}

A gestão governamental aberta é a chave para alcançar a transparência e a confiança da população nas instituições públicas, o que impede que as informações geradas sejam utilizadas de forma permanente e segura; facilitando o conhecimento das ações que a gestão pública vem desenvolvendo. O objetivo é examinar e analisar as evidências científicas existentes sobre a gestão de governo aberto em instituições públicas, utilizando as bases de dados EBSCO, SCOPUS e SCIENCE DIRECT em inglês e espanhol nos últimos quatro anos, utilizando o termo "gestão de governo aberto", descartando artigos não referenciados e acadêmicos. Tendo como resultados que para tornar a gestão transparente nas instituições públicas, novos paradigmas devem ser desenvolvidos na gestão do governo aberto, 
começando com ações para melhorar a geração de informações para seu uso pela população, em diferentes ações de caráter social ou econômico. natureza, sem enviesar as informações, restringindo-se ao uso adequado da instituição pública. Conclui lembrando que para uma gestão governamental aberta eficiente e eficaz, devemos levar em consideração os desafios da gestão transparente com a utilização de dados abertos para uso e benefício da população, tornando transparentes as ações e recursos que a administração pública investe.

Palavras-chave: Governo aberto, dados abertos, abertura de governos públicos, gestão pública.

\section{INTRODUCCIÓN}

En los Estados Unidos los niveles de competitividad y los altos niveles de un mundo cada vez globalizado, exige que en la administración pública usen obligatoriamente herramientas de gestión para manejar la información de forma eficiente y eficaz que generan las entidades diariamente; sin embargo, las investigaciones refieren que existe una multitud de desafíos e impedimentos para la gestión del gobierno abierto, identificados en tres categorías: impedimento al acceso de datos (disponibilidad de datos y la capacidad de búsqueda), uso de datos (relacionados con problemas con impedimentos de usabilidad, comprensibilidad, calidad, compatibilidad y metadatos) y deposición de datos, es decir, dificultades en la interacción con el proveedor de datos y los desafíos para liberar los conjuntos de datos, así como los problemas de comunicación entre el usuario final y los datos de la entidad (Luna-Reyes y Najafabadi, 2019). Lo cual hace indicar que estos impedimentos hacen que la información para gestión del gobierno abierto no se trabaje en forma eficiente y eficaz.

En Europa específicamente en República Checa, la corrupción y el aumento de la desigualdad están estrechamente relacionado a la transparencia, métodos y procedimientos que ayuden en la toma de decisiones gubernamentales; siendo uno de los canales de comunicación los cabildos, que es el intercambio bidireccional de información entre los grupos de presión y los funcionarios públicos, debiendo de ser esta llevada de manera transparente (Laboutková, 2018). Es decir, los canales de comunicación ayudan con el intercambio la información de manera transparente en la gestión pública.

Ahora la región Latinoamérica presenta mayor desconfianza (32\% según datos de Latinobarómetro) en la administración pública, países como Brasil o Chile tienen altos niveles de desarrollo de la administración pública y muy poca confianza y satisfacción con los servicios públicos, la explicación de la falta de correlación entre la calidad de la burocracia y la confianza es probablemente atribuible al desarrollo histórico de la construcción de la administración pública y las reformas incompletas, los altos niveles de desigualdad del país que conspiran contra el desarrollo de una idea de ciudadanía, no hay acceso universal a los servicios públicos, ni se percibe que los procedimientos de prestación de los mismos sean justos e imparciales. Es decir, la corrupción y la desconfianza se configuran como problemas de acción colectiva (Güemes, 2019). Con ello se confirma el problema de desconfianza en la administración pública lo cual está estrechamente ligado con la corrupción y da como resultado una población insatisfecha y con desconfianza en los servicios públicos.

En el Perú, la población percibe que la corrupción es el problema principal ligada con el poder político, el tráfico de influencias, los porcentajes de cobro en las contrataciones públicas, defraudaciones, etc., con un efecto del 60.6\% (INEI, 2020); es decir, más de la mitad de la población desconfía de las labores que realizan los funcionarios públicos. A pesar que, en el Perú normó la Ley $\mathrm{N}^{\circ} 27806$ - Ley de transparencia y acceso a la información pública del 13 de julio de 2002 que tiene por finalidad promover la transparencia en los actos del Estado y regular el derecho fundamental del acceso a la información y su reglamento con Decreto Supremo N ${ }^{\circ}$ 072-2003-PCM 
del 7 de agosto de 2003 (PCM, 2002); el Decreto Supremo N 063-2010-PCM del 2 de junio de 2010 aprueba la implementación del Portal de Transparencia Estándar en las Entidades de la Administración Pública, donde establece que se faciliten los recursos para la elaboración de los accesos de transparencia estándar (PCM, 2010) y con Resolución Ministerial 035-2017-PCM se aprobó la directiva $\mathrm{N}^{\circ}$ 001-2017-PCM/SGP donde se establecen las directrices que deben tomar en cuenta las entidades estatales para la elaboración de los sitios web de transparencia estándar (PCM, 2017b); sin embargo, los esfuerzos del gobierno de transparentar la administración pública a través de la normatividad, no son percibidos ni son significativos por el resultado alto expresado en porcentaje de la corrupción que percibe la población, es decir, no ha habido avance en la disminución de la corrupción.

Sumado a ello, se tiene los trabajos de fiscalización a los sitios web de Transparencia Estándar, que arrojan resultados en los Ministerios y la Presidencia del Consejo de Ministros (19) cuyo promedio fue $83 \%$ de nivel de cumplimiento; en los gobiernos regionales (25) fue del $53 \%$ y en los gobiernos locales (25 municipalidades capitales de Departamentos) el resultado del 41\%, en el periodo 2018, específicamente el gobierno regional de San Martín obtuvo el $79 \%$ de nivel de cumplimiento situándolo en el sexto lugar a nivel de gobiernos regionales en el trabajo verificación a los sitios web de Transparencia Estándar realizada por la Defensoría del Pueblo (Defensoría del Pueblo, 2018); es decir, en el Perú en ningún nivel de gobierno se cumple con presentar la información al 100\% en la web de Transparencia Estándar.

Sin embargo, el Perú desde el año 2012 integra la Alianza para el Gobierno Abierto, con los objetivos de i) Mejorar los niveles de transparencia y rendición de cuentas de la administración pública mediante la apertura de datos; ii) Promover y expandir los mecanismos de participación ciudadana en los asuntos públicos que les conciernen; y iii) Generar la colaboración entre entidades públicas y sociedad civil para la búsqueda e implementación de soluciones a los problemas públicos (PCM, 2017a). Y para su aceptación el Perú debió elaborar el Plan de Acción conteniendo los compromisos en tema de transparencia y acceso a la información pública, integridad pública, Gobierno Electrónico y promoción del desarrollo de nuevas tecnologías, participación ciudadana y rendición de cuentas (PCM, 2018). Cumpliendo así con los requisitos para ser admitido.

En base a la política Nacional de Modernización de la Gestión Pública aprobada con decreto supremo n. ${ }^{\circ}$ 004-2013-PCM, cuya visión de Estado moderno es definido como el que está orientado al servicio del ciudadano, eficiente, unitario y descentralizado, inclusivo y abierto (transparente que rinde cuentas) (PCM, 2017a), con lo cual, entre otras, se pretende aumentar el nivel de confianza de la población hacia sus entidades públicas. La política Nacional de Modernización de la Gestión Pública es una herramienta que orienta la actuación de la administración pública en todos sus niveles de gobierno y que debe ser aplicada por los gobiernos regionales y locales incorporando el concepto de gobierno abierto, como eje transversal de la política de Modernización del Estado (PCM, 2018).

Y actualmente, el Perú cuenta con una Estrategia y modelo nacional de datos abiertos gubernamentales del Perú 2017-2021, aprobados con decreto supremo $\mathrm{N}^{\circ}$ 016-2017-PCM publicado el 10 de febrero de 2017; estando la estrategia Nacional de datos abiertos gubernamentales alineada a la Política Nacional de Modernización de la Gestión Pública; para la implementación de los datos abiertos, deben tener en consideración las entidades al momento de formular sus instrumentos de gestión, el alineamiento con las políticas, planes, estrategias y agendas nacionales de la sociedad de la información y de competitividad (PCM, 2017a). 
Por su parte, la Alianza para el Gobierno Abierto, propone la apertura de datos de las instituciones públicas a cualquier nivel para uso de su población con el fin de transparentar la administración pública y con ello la rendición de cuentas, con el cual se beneficia a la población en el uso de los recursos públicos a favor de ellos mismos, representando mayores desafíos en las entidades públicas y también demanda nuevos conocimientos.

\section{FUNDAMENTO TEÓRICO}

Para hablar de gobierno abierto primero debemos referirnos a las políticas públicas que están integradas a sistemas complejos difíciles de controlar y están continuamente sujetas a fallas por la incompetencia, la corrupción, la falta de recursos y/o la mala gobernanza; presentando las siguientes patologías: las políticas públicas son no lineales y emergentes, la no linealidad es el efecto final de una política pública y ésta produce fenómenos emergentes, cuyos resultados no se pueden predecir fácilmente, sólo pueden aclararse y entender al ejecutar el sistema (Mueller, 2019).

Asimismo, las políticas públicas no se asientan en equilibrio y son difíciles de predecir, siendo las predicciones requisitos fundamentales del enfoque estándar de las políticas públicas; las políticas públicas evolucionan y coevolucionan y están determinadas de manera crucial por la cultura, creencias, instituciones, normas y tecnología; y la coevolución tiene lugar cuando exista un cambio en un agente en evolución. Además menciona, que las políticas públicas están sujetas a sesgos cognitivos, en el entendido que si las personas son muy racionales y están sujetas a todo tipo de sesgos cognitivos y se vuelven considerablemente más difíciles de corregir. Y por último, afirma que las políticas públicas sufren de reactividad, que es el hecho que los grupos e individuos sujetos a una política a menudo son conscientes del hecho que están siendo manipulados y reaccionan a propósito, alterando el impacto de la política en el proceso (Mueller, 2019).

A lo cual, Hofmann, Sæbø, Braccini, Za (2019) señalan que, en los últimos 30 años, las organizaciones del sector público han evolucionado de estructuras rígidas y burocráticas a formas más descentralizadas de coordinación, ligado con los procesos de transformación digital que ha desafiado al sector público a responder a la evolución de una sociedad más digital, influyendo en las posiciones del valor público (responsabilidad hacia los ciudadanos, la eficiencia, la efectividad y la equidad; todo ello la introducido el concepto de nueva gobernanza pública, que da paso a las nuevas formas de prestación de servicios públicos, como las redes interorganizativas autoorganizadas que incluyen las políticas gubernamentales como las no gubernamentales.

Es así que, Santos y Santos (2017) señalan que, en los modelos teóricos de administración pública tenemos a la nueva gestión pública y la nueva gobernanza pública; siendo que la nueva gestión pública sus bases están relacionadas con los factores: centrarse en el ciudadano, orientación hacia resultados, a través de planificación estratégica e indicadores de desempeño, énfasis en la transparencia y el control social, flexibilización de la gestión, apreciación y desarrollo de los funcionarios públicos. Existiendo una relación entre el compromiso con los resultados para el ciudadano y la responsabilidad de los políticos y los funcionarios públicos, planteando la idea de efectividad que se dirige hacia organizaciones más flexibles (reducción de la burocracia), innovadoras y eficientes, cuyo enfoque principal para la prestación de servicios públicos es el ciudadano (llamado cliente) el cual recibe un trato más personalizado.

Además señalan que, la nueva gobernanza pública surge por las brechas que se generan al hacer uso de los principios comerciales del sector privado para mejorar el desempeño del gobierno, 
causada por la inconsistencia que ocurre debido a la falta de denominadores como "ganancias" o "retorno de la inversión", que son comunes en el entorno privado; y es difícil hacer una comparación porque los programas públicos se basan en la confianza de los ciudadanos en la legitimidad de sus instituciones más que en la eficiencia centrada en el mercado; la nueva gobernanza pública coloca los valores políticos en el centro del debate en oposición al enfoque estrictamente instrumental de la nueva gestión pública (Santos y Santos, 2017).

Según Aleixandre-Benavent, Ferrer y Peset (2019), existen los siguientes términos: el Open data o el acceso abierto a los datos, se relaciona con los conceptos, como open science (ciencia abierta), big data (datos masivos) y data government (datos gubernamentales). Mientras que en un concepto amplio el Open research science significa el acceso abierto a la ciencia y que comprende el open access - que viene a ser el acceso abierto a las publicaciones? y open research data ? viene a ser el acceso abierto a los datos de investigación.

Y en América Latina, Bonina y Eaton (2020) señalan que, el establecimiento de plataformas de datos abiertos en Buenos Aires, Ciudad de México y Montevideo, ciudades que cuentan con principales avances de datos de gobierno abierto (DAG), han tenido su evolución histórica en: Buenos Aires la iniciativa de datos abiertos, se lanzó formalmente en marzo de 2012, cuando el gobierno aprobó el Decreto de Gobierno Abierto 156/2012. El Decreto presentó pautas para una política basada en la participación ciudadana, la transparencia y la colaboración. El decreto fue un factor clave para permitir la creación de la Oficina de Innovación y Gobierno Abierto dentro del Ministerio de Modernización.

Luego en la Ciudad de México el primer intento de establecer un portal de datos abiertos fue en 2012 y la Oficina Administrativa lanzó una plataforma de DAG para facilitar el acceso a los datos obligatorios según lo requerido en la Ley de Libertad de Información de México (FOI). Hubo poco apoyo para esta plataforma y el interés se desvaneció. Una actividad alternativa de DAG surgió en paralelo en 2013 cuando el gobierno de la ciudad lanzó el Laboratorio para la Ciudad que reunió a un equipo multidisciplinario de diseñadores, científicos sociales, arquitectos, expertos en tecnología y formuladores de políticas para fomentar proyectos urbanos innovadores y se convirtió en el coordinador del programa de Gobierno Abierto de la ciudad en general. Junto con cuatro secretarios del gobierno y una organización no gubernamental, tenía el mandato de liderar el desarrollo de las políticas de datos abiertos en la ciudad. Esto incluyó el lanzamiento de una nueva plataforma de datos de gobierno abierto para la cual asumiría la propiedad. Para facilitar este proceso de arranque, el Laboratorio desarrolló una herramienta social en forma de una iniciativa llamada "Código para la Ciudad de México" en junio de 2013 (Bonina y Eaton, 2020).

Y por último, en la capital uruguaya de Montevideo fue la primera ciudad de América Latina en tener una política de datos abiertos. Su plataforma DAG comenzó como un movimiento ascendente entre un pequeño grupo de servidores públicos de nivel medio en 2009. Posteriormente, se creó un Grupo de Trabajo de Datos Abiertos informalmente dentro del gobierno y comenzó a incorporar datos abiertos en la agenda del gobierno de la ciudad. Más tarde convenció a las autoridades de la Ciudad de aprobar la Resolución Municipal 640/10 en febrero de 2010 para establecer una política de datos abiertos de manera más formal. La Resolución de datos abiertos de Montevideo estableció un compromiso del gobierno para publicar datos abiertos y establecer reglas específicas para publicar los datos en formato abierto. La Resolución también estableció un grupo de trabajo para dirigir la publicación de datos abiertos en un portal (datos.montevideo.gob.uy) alojado por el Departamento de Tecnología y Desarrollo de Software (Bonina y Eaton, 2020). Pero, Copaja-Alegre y Esponda-Alva (2019), señalan que no puede limitarse el uso de las Tecnologías y 
Comunicación (TIC) a la gestión abierta y participativa, esta debe incluir políticas de inclusión digital a fin de evitar un aumento en la segregación social.

Los orígenes de gobierno abierto datan de 1957 en Estados Unidos donde aparece por primera vez la noción open government, en el artículo "The open government principle: Applying the right to know under the Constitution", publicado por Wallace Parks (1957), como parte de un proyecto del Sub- comité Especial sobre la Información del Gobierno del Congreso de Estados Unidos para la elaboración de la Ley de Libertad de Información (Freedom of Information Act, FOIA); y por 1970, en el Reino Unido, el término gobierno abierto es utilizado en iniciativas protransparencia, como el movimiento para "abrir las ventanas" del sector público hacia el escrutinio ciudadano. (Figueras, 2019).

Y desde el 2009, está ligado el gobierno abierto a la transparencia cuando el Presidente de Estados Unidos, Barak Obama, promulgó el "Memorando sobre Transparencia y Gobierno Abierto"; constituyendo esta acción como un referente para diversas iniciativas sobre gobierno abierto alrededor del mundo. La noción de gobierno abierto aparece hoy en el ámbito académico como un tema de vanguardia asociado con la reforma gubernamental y la promoción de la democracia deliberativa, aquella que considera la promoción del desacuerdo deliberativo, del debate público razonado, como la base del entendimiento para construir políticas públicas con reciprocidad, publicidad y rendición de cuentas (Figueras, 2019).

Por lo que, Hudson y Florez (2019) consideran que el Gobierno abierto, se ha constituido como un mecanismo para que los pobladores mejoren su calidad de vida, éste constituye un gran desafió, porque los recursos estatales representan un hito notable de observar y es por ello que deben contar con metodologías confiables a implantar para realizar la vigilancia del destino del capital público, de tal forma la transparencia y la participación forme parte del panorama institucional.

Mientras, Gil-Garcia, Gasco-Hernandez, Pardo (2020), señalan que, a pesar de algunos avances significativos en la investigación y algunas implementaciones exitosas en el mundo real, la velocidad con la que estos eventos se han desarrollado y la "necesitad" implementar proyectos de gobierno abierto debido a presiones nacionales o internacionales ha producido cierta confusión y ambigüedad. También hay diversidad en la conceptualización del gobierno abierto y cómo afecta iniciativas o estrategias específicas. De hecho, aunque muchas iniciativas han incluido datos abiertos y toma de decisiones abierta, los gobiernos generalmente han perseguido diferentes prioridades y estrategias de implementación, que incluyen varias dimensiones del gobierno abierto y como resultado, el desarrollo del gobierno abierto en todo el mundo ha sido muy diverso, sin ningún acuerdo sobre las dimensiones, elementos o componentes de gobierno abierto.

Veres (2020), señala que una de las tendencias para mejorar la calidad de la información, es la filosofía Lean Management que plantea la reducción de existencias, reducción de costos, reducción de espacio, reducción de riesgos, reducción de desperdicios, racionalización de procesos, aumento en la productividad, mejora de calidad, aumento de satisfacción de clientes. Siendo el mayor desafío la implementación de gestión y desarrollo de un modelo conceptual por la resistencia humana a cambio, pero su aplicación mejora la percepción en el lugar de trabajo, mayor productividad, mejor organización y claridad de procedimientos.

Otra tendencia para mejorar la calidad de información según Widjajarto, Lubis, Yunan (2019) es el desarrollo del modelo arquitectónico de infraestructura de tecnología de la información 
basada en la calidad del servicio en instituciones gubernamentales, utiliza la base de una metodología de colaboración abierta con la consideración de los usuarios de la aplicación o usuarios internos y externos a la organización, es el principal y determinante final del nivel de satisfacción del servicio. Se define como innovación con proyectos orientados a la producción, donde los participantes están coordinados de manera flexible para generar servicios para contribuyentes o no contribuyentes directamente. El uso de datos de satisfacción proviene de usuarios externos de infraestructura y partes internas de infraestructura.

Y Cadena (2019) señala que, se debe tener en cuenta que la Web de una organización pública contará con información para ser utilizada por las empresas y ciudadanos llamados reutilizadores, siendo la falta de la calidad de información publicada una barrera para que el proceso se reutilización sea masivo. Así los datos que se crean, almacenan, procesan, intercambian, comparten, agregan y reutilizan, son de calidad si son útiles, es decir, si se obtiene valor de esperado y para asegurar la calidad de los datos en una organización se requiere: i) tener en cuenta las necesidades de los usuarios; ii) definir un proceso de generación de datos claros; iii) identificar el ciclo de vida de los datos; iv) nombrar a una persona que gestione los datos y v) evaluar la calidad del producto de datos.

Pero el esfuerzo que realizan las organizaciones y la administración pública para publicar los datos abiertos se ve afectado cuando no existe una gestión adecuada de la calidad de los datos abiertos publicados en su portal, ocasionando que se desechen al poco tiempo de ponerse en marcha. Por ello, en un portal web la calidad de los datos debe ser un proceso multidimensional que se inicia desde el momento que se digitaliza la información y considera los requisitos para su reutilización, según las siguientes dimensiones:

1. Exactitud, es decir, datos que representan correctamente la entidad o evento del mundo real.

2. Puntualidad, datos que representen la situación real y la agilidad en la publicación.

3. Consistencia, no contienen contradicciones.

4. Exhaustividad, incluyen todos los elementos de datos que representan a la entidad o evento.

5. Disponibilidad, accesibles ahora y con el tiempo.

6. Conformidad, siguen las normas aceptadas.

7. Credibilidad, son fuentes confiables.

8. Accesibilidad, son legibles por máquina.

9. Relevancia, cantidad adecuada de datos.

Siendo que las cuatro primeras dimensiones de calidad dependen de los sistemas de información de origen, y las dimensiones de la 5 a la 9 dependen de la gestión implementada en los portales de datos (Cadena, 2019).

Por otra parte, la apertura de gobiernos se formalizó a través del lanzamiento multilateral del Open Government Partnership (OGP) en 2011 con la participación de ocho gobiernos: Brasil, Indonesia, Filipinas, México, Noruega, Sudáfrica, Reino Unido y Estados Unidos y actualmente se ha expandido a 75 gobiernos nacionales y 15 gobiernos subnacionales (regionales, provinciales y locales) siguiendo su expansión en curso. Donde académicos, políticos, responsables políticos y 
profesionales buscan información sobre cómo las reformas de gobierno abierto pueden conducir a mejorar sosteniblemente la vida de las personas (Chatwin, Arku y Cleave, 2019).

\section{PROCEDIMIENTOS METODOLOGICOS}

Se efectuó una exploración sistemática, la cual fue elaborada por estudiantes del doctorado en Gestión Pública y Gobernabilidad de la Escuela de Postgrado de la Universidad César Vallejo, Perú. Cuya obtención de los datos se ejecutó en cuatro meses aproximadamente, realizando una revisión del grado de implementación de la gestión de gobierno abierto en el mundo, abarcando artículos científicos de distintos países como: medio oriente, países bajos, países de América latina, Estados Unidos, Corea del Sur y Reino Unido.

Dentro de los criterios de selección tenemos a tipos de intervinientes donde se incorporaron estudios de literatura de ámbito teórico. Tipos de estudios: tomando en cuenta que existe un número amplio de estudios sobre gobierno abierto en el mundo esta investigación tuvo como objeto elaborar un mapa de los conocimientos existente e identificar su relevancia. Tipos de resultados: Se seleccionaron como resultados primarios a las publicaciones originales de estudios científicos.

Se realizó la búsqueda directamente en la base de datos de EBSCO, Scopus y Science Direct, realizando el filtrado referente a: publicaciones arbitradas y académicas, en el rango de tiempo desde el 2017 al 2020. Selección de estudios: fue realizado la selección de los estudios por dos revisores independientes y cualquier controversia fue resuelta por un tercer revisor. Para la selección de los artículos se utilizaron cuatro criterios: 1) artículos que incluyan las palabras "gobierno abierto" o "datos abiertos" en el título, resumen o palabras claves; 2) artículos que incluyan la palabra "gestión" o "gestión pública" en el título, resumen o palabras claves; 3) artículos que incluyan las palabras "apertura de gobiernos" o "apertura de los gobiernos públicos"; 4) artículos cuyo ámbito de estudio sea instituciones públicas; para posteriormente revisar los artículos completamente para confirmar si son elegibles o no.

La búsqueda dio como resultado 1208 referencias con nuestros criterios se descartaron 1102 referencias que no cumplieran con contener las palabras en el título, resumen o palabras claves, culminado este primer proceso para realizar la selección se procedió a la lectura de 106 citas para contrastar la selección realizada. En este proceso de lectura y revisión de las 106 citas se descartaron 78 artículos o estudios que no cumplían con los criterios para incluirlos, quedando 28 artículos, que luego una revisión minuciosa se descartaron 10 artículos porque no cumplieron los criterios de inclusión. Terminando este proceso, se obtuvo 18 artículos científicos, que se presentan de manera sistemática y descriptica, porque la naturaleza de estos estudios no permite ningún otro tipo de análisis de igual manera (Moher et al., 2009).

Los aportes o propuestas se categorizaron y agruparon en función a los pilares de gobierno abierto, tal como Macías, Valencia, Maldonado y Brito (2020) señalan que la gestión de gobierno abierto se fundamenta en los pilares de gobierno transparente, colaborativo y participativo y estos son los medios para lograr una mayor democracia con lo cual se crea las condiciones para aumentar los rendimientos del gasto público; para lograr su implementación se necesitan herramientas de gestión con la cual se pueda evaluar y capacitar a la ciudadanía con el fin de incentivar una cultura de transparencia, participación, acceso a la información y colaboración, en este sentido para exponer y discutir los resultados del procesamiento de los datos se compararon los artículos, a fin de determinar los principales cambios y estándares a aplicar para mejorar la gestión pública en base a la herramienta de gobierno abierto, mediante el cual se hacen inferencias, deducciones y análisis de los artículos y trabajos seleccionados para su valoración.

\section{RESULTADOS Y DISCUSIÓN}




\subsection{Resultados}

Para Macías, Valencia, Maldonado y Brito (2020) la gestión de gobierno abierto se fundamenta en los pilares de gobierno transparente, colaborativo y participativo y estos son los medios para lograr una mayor democracia con lo cual se crea las condiciones para aumentar los rendimientos del gasto público; para lograr su implementación se necesitan herramientas de gestión con la cual se pueda evaluar y capacitar a la ciudadanía con el fin de promover una cultura de transparencia, acceso a la información, participación y colaboración.

Pero el gobierno abierto presenta dimensiones institucionales (como son la política y estrategia, fundamentos legislativos, arreglos organizativos, habilidades relevantes y apoyo educativo, apoyo y conciencia pública) las cuales nos son estáticas sino dinámicas y deben ser consideradas por los responsables de la formulación de las políticas (Safarov, 2020); que corresponden ser analizadas a través de sus indicadores, para lo cual es necesario la aplicación de herramientas como el uso de un marco de criterios y subcriterios (responsabilidad, transparencia, participación, eficacia, igualdad, sostenibilidad, visión y planificación, legitimidad y burocracia, capacidad cívica, prestación de servicios, economía eficiente, relaciones y seguridad), se puede llegar a medir los servicios a fin de lograr la universalidad, la comparabilidad y la clasificación de los servicios en las áreas geográficas u organizaciones de gobierno; con la finalidad de evaluar la calidad de la gobernanza (Biswas, Jana, Arya y Ramamritham, 2019).

Actualmente, se tiene que el antiguo gobierno abierto se refiere a la libertad de información disponible para los ciudadanos para que las autoridades gubernamentales sean transparentes y responsables, resaltando la contribución de las leyes de libertad de información para la mejora de la transparencia, la responsabilidad y la confianza pública; pero, este enfoque tradicional se reconsideró y reformuló cuando las tecnologías web ofrecieron nuevos canales a través de los cuales se podría proporcionar más información simultáneamente a varias personas no identificadas más allá de las limitaciones de tiempo y espacio. Logrando que las iniciativas de gobierno abierto se enmarquen en la reutilización de la información pública, así como el suministro proactivo de información pública antes que lo pidan ciudadanos; es decir, ha habido cambios dramáticos en muchos aspectos al variar el enfoque convencional de libertad de información para los ciudadanos (Moon, 2020).

Existen debilidades en la implementación de nuevas estrategias basadas en el gobierno abierto, según Alban y España (2019), debido a que éste debe ser concebido como una política de estado para que sean efectivos y duraderos sus resultados; es decir, debe partir de la voluntad política de sus gobernantes para el impulso e implementación de éstas nuevas estrategias como la formación y sensibilización de sus funcionarios públicos, la ciudadanía, el sector privado y todos los actores e involucrados.

Mientras que Matheus y Janssen (2020), en su investigación denominada: Un estudio sistemático de literatura para desentrañar la transparencia habilitado por los datos de gobierno abierto: la teoría de la ventana; señala que la teoría de la ventana es un modelo unificador que contiene determinantes (agrupado en cuatro grupos de factores: calidad de datos, calidad del sistema, características organizacionales y características individuales y efectos de la transparencia), es decir, unifica los diversos trabajos en esta área que se originan en la administración pública y las publicaciones del sistema de información; siendo probable que la naturaleza y los objetivos de la transparencia difieran según la situación y el modelo se puede utilizar para seleccionar los determinantes que sean apropiados para un contexto dado. 
A esto suma Schnell (2020), en su estudio: Visión, voz y tecnología: ¿Existe una tendencia global de "Gobierno Abierto?, donde identifica dos interpretaciones conectadas pero algo diferentes de la apertura gubernamental: una estrecha, que se centra en herramientas para aumentar la transparencia y la participación en el gobierno, y una amplia en la que los gobiernos son sistemáticamente transparentes y abiertos a la participación de los ciudadanos. La primera interpretación, estrecha, es particularmente compatible con el énfasis tecnológico del discurso y la práctica de la "nueva" apertura. La segunda interpretación, más amplia, reconoce más explícitamente la dimensión política y basada en los derechos de la apertura.

Tal es así que, Gonzálvez-Gallego, Nieto-Torrejón y Pérez-Cárceles (2020), señalan que la relación entre los datos abiertos del gobierno y la confianza está influenciada por la satisfacción y que los ciudadanos pueden acceder a datos públicos relacionados con el pasado, cuando se sienten más satisfechos y es donde esta satisfacción se proyecta hacia el futuro aumentando la confianza en las instituciones públicas; lo cual permite a los ciudadanos que a través de los datos abiertos monitorear el desempeño del gobierno y su gestión, lo que aumenta su satisfacción con la calidad de la democracia y los servicios públicos.

Y por su parte Sandoval-Almazan (2019) refiere que existe cumplimiento a la ley por la mayoría de los estados, pero esto no quiere decir que sean más transparentes o sus prácticas se acerquen más al gobierno abierto, sino que permiten el acceso abierto a cierta información gubernamental, es decir cumplen a nivel básico. Por lo tanto, las políticas públicas se deben llevar más allá de las leyes y reglamentos de tal forma que permitan impulsar prácticas de gobierno abierto en las instituciones públicas en todos los niveles de la administración pública.

Ibarra, Mballa, Ibarra y Vega (2019), refiere que las diferentes formas de gobierno y los mecanismos para elaborar las políticas públicas plantea la necesidad de definiciones conceptuales que incluyan la articulación y la participación de los diferentes involucrados en los procesos de solución de los problemas de la administración pública, para lograr un gobierno más pluralista y cooperativo desde un gobierno centralizado y se requiere replantear el marco interpretativo del gobierno local, con atención en los actores y sus acciones y es donde propuesta de modelo de gestión abierta de forma conceptual tiene acogida.

La colaboración es el conjunto de contribuciones ciudadanas sustentadas en principios basados en mejorar la calidad de vida de la población y significa la actitud y disposición de hacer las cosas (en sus dimensiones política, económica, social y cultural), teniendo sustento en la relación gobierno abierto y gobernanza, siendo el primero los cambios en la organización y en la gestión pública, para posibilitar afianzar el modelo de la gobernanza frente a las expectativas crecientes de los ciudadanos en la forma como el gobierno atiende sus necesidades en el marco de la Posnueva gestión pública (Chica-Vélez y Salazar-Ortiz, 2020).

Actualmente, el gobierno público debe considerarse como una organización que puede estimular, promover y facilitar el espíritu empresarial y la innovación en los ecosistemas de ciudades inteligentes al ser más colaborativo a medida que el mercado se expande más allá de su concepto original; para ello debe identificar las barreras como: ausencia de reglas, tareas y responsabilidad, visión insuficientemente integrada del urbanismo, insuficiente adecuación de estilos administrativos y coordinación y comunicación interdepartamental, aversión al riesgo, disponibilidad de datos, desincentivos y normas de contratación pública no flexibles, escasos 
recursos, la inadecuada capacidad tecnológica; lo cual es útil para comprender cómo superar ellos, con el fin de fomentar prácticas de gestión, innovación y emprendimiento en los ecosistemas locales (Ferraris, Santoro y Pellicelli, 2020).

Si bien la transparencia es el elemento más asociado al término Gobierno Abierto y representa una tendencia en la gestión y políticas públicas, los resultados de su estudio RuvalcabaGomez y Renteria (2020) permiten inferir que el sector de las organizaciones de la sociedad civil (OSC) tiene críticas al gobierno por sus políticas de transparencia activa y proactiva y la brecha de éxito en la percepción de los dos sectores (gobierno y sociedad civil) representa una evidencia que se debe considerar para fortalecer el diálogo entre sociedad civil y gobierno para co-crear políticas de transparencia y acceso a la información. Puesto que la participación ciudadana avanza hacia la consolidación de una práctica institucional pero existe un avance muy pobre desde la perspectiva de la OSC, mientras que la perspectiva del gobierno es mucho más positivista sobre el tema.

El uso de datos abiertos y la participación pública, según refieren Lassinantti, Ståhlbröst y Runardotter (2019), es un proceso muy heterogéneo, lo cual además conduce a diferentes formas de compromiso y participación relacionada con las tareas públicas, que abarcan el conocimiento de problema y diferentes formas de personas con conocimientos en áreas de aplicación comparados con personas con habilidades en técnicas de datos. Tal es así que, Royo-Montañés y Benítez-Gómez (2019), señalan que existe mucho por avanzar, tanto en la adopción de portales de datos abiertos, como en el desarrollo de los ya existentes, fomentando la utilización de los datos por parte de un público no experto (como tutoriales, secciones de ayuda o demostradores), la rendición de cuentas, la participación ciudadana, y la calidad de los datos suministrados; es decir, que el gobierno abierto se sustenta en el diálogo entre el gobierno y los ciudadanos, en esta filosofía se encuentran enmarcada sus iniciativas; por lo tanto, las demandas e intereses de la población no se deben desatender.

Por su parte, Purwanto, Zuiderwijk y Janssen (2020) señalan que, la participación ciudadana con datos abiertos gubernamentales puede verse influida positiva o negativamente por diferentes factores, como factores personales, relacionados con el desempeño, económicos, técnicos, sociales y políticos; y que el modelo postulaba que los perfiles de los ciudadanos como edad, género, nivel educativo, capacidades, competencia, recursos, conciencia, experiencia y voluntariado podrían afectar la solidez de esta relación, lo cual se puede utilizar para analizar los determinantes de las intenciones de los ciudadanos de comprometerse con datos abiertos gubernamentales y los factores moderadores que influyen en la relación entre los determinantes y las intenciones. Zuiderwijk, Shinde y Janssen (2018) señalan que existen las iniciativas de datos de gobierno abierto cuyos objetivos van desde mejorar la transparencia, la responsabilidad, aumentar la innovación y la participación los cuales no están alineadas con sus beneficios como son mayor apertura, confianza o innovación, pero con la ayuda de otras iniciativas se puede lograr pero la inversión y el esfuerzo son alto, resultando que éstos objetivos se cumplen mayormente a nivel nacional que en los diferentes niveles de gobierno a nivel local y regional.

Debe tenerse en cuenta que la nueva gestión pública también está ligado al nuevo gobierno abierto, por lo cual (Moon, 2020) realiza una comparación entre dos enfoques el viejo gobierno abierto y el nuevo gobierno abierto a través de cuatro dimensiones:

1. Dimensión política que está representada por la libertad de información versus la política de gobierno abierto; en la cual existe la controversia sobre la información mostrada al público, puesto que choca con los mandatos de protección como la privacidad y la seguridad 
nacional, si es perjudicial para los interés públicos, el gobierno debe segregarlo del resto de información

2. Dimensión de información y datos, que se refiere a la información tradicional versus a los datos digitalizados conectables y reutilizables; donde la calidad de datos se ve perjudicada por las operaciones burocráticas, así como la resistencia y renuencia a transformar los datos legibles a datos conectables y reutilizables.

3. Dimensión de valor administrativo, referido a la transparencia versus la interactividad (participación y colaboración); con el cambio de la política tradicional de libertar de información hacia las iniciativas de gobierno abierto, no se considera a la transparencia con un valor intrínseco, pero si para estimular la participación ciudadana y el gobierno colaborativo, donde los ciudadanos tienes roles activos como co-decisión en las políticas públicas.

4. Dimensión ciudadana, referida a los usuarios y productores de información versus activos; a pesar que los ciudadanos tienen un papel más activo en las políticas públicas, existe una brecha digital que limita la participación de los ciudadanos de escasos recursos o que tienen menos conocimientos con el uso de la tecnología lo que limita a participar en los asuntos públicos, lo cual es un preocupación no solo por la accesibilidad de la información pública sino por sus utilidades y beneficios de la información pública.

Por otra parte, Chatwin, Arku y Cleave (2019) señalan que las políticas públicas se encuentran establecidas por el gobierno nacional las cuales deben facilitar su transferencia entre sus jurisdicciones y es evidente que en cualquier área específica ésta no avance y no se desarrolle en el contexto local; además que, la Alianza de Gobierno Abierto muestra una agrupación específica de políticas y no está claro si ésta representa la combinación óptima de políticas que permiten implementar un gobierno abierto, un ejercicio de la conveniencia política y facilidad para el desarrollo de políticas, corriendo el riesgo de que las iniciativas de gobierno abierto no se enrumben a la eficiencia y eficacia que se quiere lograr. De ahí radica la importancia que la política pública considere el contorno local es por ello que Open Government Partnership (OGP) impulsó a los gobiernos subnacionales ser participe en enfatizar los aspectos del gobierno abierto para así abordar sus necesidades, de forma tal que permita adaptarse a los diferentes contextos geográficos; donde la participación, la rendición de cuentas y la transparencia sigue siendo importantes y que a través del alcance de la implementación y el impacto de los planes de acción para abrir la información y procesos relevantes constituye el primer paso para la recopilación de las mejores prácticas de las iniciativas de participación ciudadana.

\subsection{Discusión}

La gestión del gobierno abierto tiene sus bases en los pilares de Gobierno Transparente, Gobierno colaborativo, Gobierno Participativo, así tenemos:

Gobierno Transparente

La gestión de gobierno abierto es un mecanismo mediante el cual se requiere llegar a lograr un gobierno transparente, esto nos da como resultado el fortalecer la gobernanza de las instituciones públicas, cuya imagen de credibilidad en las últimas décadas se ha visto afectado; de tal forma que la población no respalda el trabajo efectuado o tiene muy poca confianza en sus instituciones públicas todo ello por los escándalos de corrupción que constantemente se han venido dando; por lo que el pilar de transparencia necesita un especial énfasis para revertir esta situación adversa, es ahí donde la aplicación de gobierno abierto cobra relevancia y no sólo desde un punto de vista de rendición de cuentas, declaraciones juradas de ingresos, publicación de gastos, sino que los datos publicados puedan ser reutilizados por la población y que sus políticas públicas estén orientadas 
específicamente a territorio a través de los gobiernos sub nacionales, para lograr de esta forma que las soluciones planteadas con la políticas públicas sean de acuerdo al contexto territorial o realidad de la población y así cerrar las brechas de infraestructura, saneamiento, etc., para la satisfacción de la población.

\section{Gobierno Colaborativo}

Más allá de contar con un gobierno transparente, para que la gestión del gobierno abierto logre involucrar a diferentes actores, es necesario que desde un marco de gobierno local se articulen y promueva la participación de diferentes involucrados donde se promueva la innovación y el emprendimiento; para lograr que el gobierno centralizado a través de diferentes procesos logre la solución de problemas en la administración pública y es como se plantea lograr un gobierno más pluralista y cooperativo. Donde la colaboración tenga como objetivo mejorar la calidad de vida de los ciudadanos, pero para ello desde el ámbito político, económico, social y cultural; debe cambiarse de actitud y tener buena disposición para hacer las cosas, con ello las expectativas de los ciudadanos de la atención de sus necesidades sea realizada por el gobierno en forma oportuna, eficiente y eficaz.

\section{Gobierno Participativo}

Un gobierno participativo es aquel donde se espera que la población participe, pero ésta muchas veces se ve limitada debido a la falta de capacitación, la edad y el acceso al internet, lo cual debe ser tomado en cuenta al momento de la creación de una política pública, su implementación, su aplicación y la evaluación de sus resultados; para lograr una verdadera participación de la población y es por ello que una política pública debe estar de acuerdo al contexto local, geográfico o realidad local y es donde los gobiernos sub nacionales recobran importancia, porque al plantear una política pública se requiere que esta sea aceptada y entendida por la población para poder contar posteriormente con indicadores de resultados no sólo en papel sino también que logren verdaderamente la satisfacción de la población a través del uso de sus servicios públicos en infraestructura hospitalaria, educativa, saneamiento, etc., Por ello, es crucial que los gestores públicos puedan tomar en cuenta dichas necesidades en el contexto local y no sólo para cumplir con un mandato del gobierno de turno, sino que su desarrollo logre involucrar a los ciudadanos y esta participación de la población junto con los pilares de transparencia y colaboración lograrán consolidar la gestión de gobierno abierto.

\section{CONCLUSIÓN}

En función a lo expuesto y tomando en cuenta los pilares de la gestión de gobierno abierto en la transparencia, colaboración y participación, podemos concluir que es fundamental reconocer la importancia innegable que tiene el gobierno abierto en las instituciones públicas; desde el enfoque político el gobierno abierto es un conjunto de reformas para gobernabilidad democrática con transparencia de la información, participación pública y rendición de cuentas, para lo cual las políticas públicas tienen que elaborarse cuidadosamente, para no correr el riesgo de convertirse en implementaciones meramente superficiales y simbólicas, pudiendo ser reformas sustanciales de mejorar los gobiernos locales abordando el contexto local y sus problemas.

En lo concerniente a las competencias analizadas en la gestión del gobierno abierto, éste debe ir de la mano con enfoques estratégicos de las políticas públicas para con ello mejorar y transparentar la gestión en las instituciones públicas; donde se enfatice en los gobiernos subnacionales con sus planes de acción de gobierno abierto que corresponden a las realidades de 
cada jurisdicción permitiendo de esta forma que las políticas públicas se alineen para lograr la implementación exitosa del gobierno abierto.

Es necesario tomar en cuenta que la calidad de los datos gubernamentales abiertos es un proceso continuo de mejora, basado en estándares y buenas prácticas, que van desde los datos que se gestionan en la entidad hasta su proceso de automatización considerando el uso de formatos abiertos. Es decir, la organización y definición de requisitos relacionado con los datos abiertos, la adaptación a estándares, además de las responsabilidades y roles que conlleva un proceso de apertura que permite mejorar la calidad de los datos publicados. En cuanto a los aspectos teóricos de la investigación, que el gobierno abierto es el protagonista dentro de administración pública para transparentar la gestión y éste se enfrenta a nuevos retos y cambios cada día más acelerados, lo que hace que tenga de adecuarse a un entorno competitivo, lo que ha significado que muchos cambios estén sin un rumbo fijo en cuanto a sus competencias, pero sin quedar rezagados y ello implica profundizar en mayor investigación.

Cabe precisar que existen algunas limitaciones en la investigación, que pueden ser consideradas para futuras investigaciones. La primera, referida a que la mayoría de artículos científicos revisados han sido seleccionados desde el punto de vista tecnocrático y muy poco, sobre el proceso de gestión de la administración pública, ni participación de gestores públicos que tienen mucha relación para lograr una apertura gubernamental; en este sentido se puede realizar estudios empíricos que permitan comprender la importancia de la participación de los gestores públicos en la gestión de gobierno abierto. La segunda limitación se relaciona con la selección de artículos científicos enfocados sólo a tres pilares de gobierno abierto, pudiéndose explorar un cuarto pilar que es la reutilización de la información pública, que con el avance exponencial del uso de las tecnologías de la información y comunicaciones han dado otro significado a la apertura gubernamental, lo cual permite establecer un enfoque multidisciplinario con la participación de las partes interesadas y los organismos de la sociedad civil. Y como tercera limitación es la selección de artículos donde se trató de incluir a todas las áreas geográficas, teniendo mayor inclinación el continente americano, por ello sería importante incorporar estudios del continente europeo, debido a su desarrollo podría fortalecer la gestión de gobierno abierto con nuevos paradigmas.

En suma, los distintos significados de los pilares de gobierno abierto y sus generalizaciones, desarrollados no deberían caer en controversia en la práctica de las investigaciones, sino representa un aporte para conocer las diferentes visiones de la ciencia y la realidad como riqueza conceptual y metodológica lo cual contribuirá a que las futuras investigación cuenten con una base para su desarrollo en temas de gobierno abierto y para finalizar, la investigación puede servir como punto de partida para seguir indagando sobre las competencias de la gestión de gobierno abierto en los gobiernos subnacionales, agregando y profundizando en algunos términos, a fin de encaminar y mejorar la gestión de gobierno abierto y realizar aportes al contenido investigado.

\section{REFERENCIAS}

Alban, H. y España, M. (2019). Debilidades en la implementación de nuevas estrategias basadas en el gobierno abierto. Dilemas Contemporáneos: Educación, Política y Valores, 1(4), 1-21. https://doi.org/10.46377/dilemas.v31i1.1022

Aleixandre-Benavent, R., Ferre, A. y Peser, F. (2019). Compartir los recursos útiles para la investigación: datos abiertos (open data). Educación Médica, xx. https://doi.org/10.1016/j.edumed.2019.07.004

Biswas, R., Jana, A., Arya, K. y Ramamritham, K. (2019). A good-governance framework for urban management. Journal of Urban Management Journal, 8(2), 225-236. https://doi.org/10.1016/j.jum.2018.12.009 
Bonina, C. y Eaton, B. (2020). Cultivating open government data platform ecosystems through governance: Lessons from Buenos Aires, Mexico City and Montevideo. Government Information Quarterly, 37(3), 101479. https://doi.org/10.1016/j.giq.2020.101479

Cadena, S. (2019). Marco de referencia para la publicación de datos abiertos comprensibles basados en estándares de calidad. http://rua.ua.es/dspace/handle/10045/102507.

Chatwin, M., Arku, E. y Cleave, E. (2019). Defining subnational open government : does local context influence policy and practice ? Policy Sciences, 0123456789. https://doi.org/10.1007/s11077-018-09347-7

Chica-Vélez, S. y Salazar-Ortiz, C. (2020). Posnueva gestión pública, gobernanza e innovación. Tres conceptos en torno a una forma de organización y gestión de lo público. OPERA, 28(28), 17-51. https://doi.org/10.18601/16578651.n28.02

Copaja-Alegre, M. y Esponda-Alva, C. (2019). Tecnología e innovación hacia la ciudad inteligente. Avances, perspectivas y desafíos. Bitácora Urbano Territorial, 29(2), 59-70. https://doi.org/10.15446/bitacora.v29n2.68333

Defensoría del Pueblo. (2018). Segundo Reporte y Balance Anual 2018: Supervisión a los Portales de Transparencia Estándar de los Ministerios del Poder Ejecutivo, Gobiernos Regionales y Municipalidades Provinciales ubicadas en capitales de departamento. 1-52. https://www.defensoria.gob.pe/wp-content/uploads/2019/04/SegundoReporte-y-Balance-Anual-2018-PTE.pdf.

Ferraris, A., Santoro, G. y Pellicelli, A. (2020). “Openness” of public governments in smart cities: removing the barriers for innovation and entrepreneurship. International Entrepreneurship and Management Journal, 16(4), 1259-1280. https://doi.org/10.1007/s11365-020-00651-4

Figueras, V. (2019). Gobierno Abierto en México : hacia una discusión realista de su factibilidad Towards a Realistic Discussion of its Feasibility. 523-554. https://doi.org/10.22201/fcpys.2448492xe.2019.235.59021

Gil-Garcia, J., Gasco-Hernandez, M. y Pardo, T. (2020). Beyond Transparency, Participation, and Collaboration? A Reflection on the Dimensions of Open Government. Public Performance \& Management Review, 43(3), 483502. https://doi.org/10.1080/15309576.2020.1734726

Gonzálvez-Gallego, N., Nieto-Torrejón, L. y Pérez-Cárceles, M. (2020). Is Open Data an Enabler for Trust? Exploring the Link and the Mediating Role of Citizen Satisfaction. International Journal of Public Administration, 43(14), 1218-1227. https://doi.org/10.1080/01900692.2019.1668412

Güemes, C. (2019). Wish you were here trust in public administration in Latin America. Revista de Administracao Publica, 53(6), 1067-1090. https://doi.org/10.1590/0034-761220180230x

Hofmann, S., Sæbø, Ø., Maria, A. y Za, S. (2019). The public sector's roles in the sharing economy and the implications for public values. Government Information Quarterly, 36(4), 101399. https://doi.org/10.1016/j.giq.2019.101399

Hudson, A. y Florez, J. (2019). Avances recientes hacia una gobernanza fiscal abierta en América Latina. Nóesis. Revista de Ciencias Sociales y Humanidades, 28(56), 1-20. https://doi.org/10.20983/noesis.2019.2.2

Ibarra, A., Mballa, L., Ibarra, M. y Vega Campos, M. (2019). Hacia Una Reconceptualización Teórica Y Metodológica Del Gobierno Abierto: Paradigma Explicativo De La Gobernabilidad Local. European Scientific Journal ESJ, 15(8), 1-24. https://doi.org/10.19044/esj.2019.v15n8p1

Instituto Nacional de Estadística e Informatíca, INEI. (2020). Perú: Percepción ciudadana sobre gobernabilidad, democracia y confianza en las instituciones. INEI, 1-43.

http://m.inei.gob.pe/media/MenuRecursivo/boletines/informe_de_gobernabilidad_may2020.pdf.

Laboutková, Š. (2018). Open government partnership: unutilized potential in post-communist EU members? (Case of the Czech Republic). Innovation: The European Journal of Social Science Research, 31(3), 350-376. https://doi.org/10.1080/13511610.2017.1415803

Lassinantti, J., Ståhlbröst, A. y Runardotter, M. (2019). Relevant social groups for open data use and engagement. Government Information Quarterly, 36(1), 98-111. https://doi.org/10.1016/j.giq.2018.11.001

Luna-Reyes, L. y Najafabadi, M. (2019). The US open data initiative: The road ahead. Information Polity, 24(2), 163182. https://doi.org/10.3233/IP-180106

Macías, J., Valencia, V., Maldonado, R. y Brito, B. (2020). Avance en los pilares del gobierno abierto, un análisis comparado. Dilemas Contemporáneos: Educación, Política y Valores, 53(1), 1-9. https://doi.org/10.46377/dilemas.v33i1.2118

Matheus, R. y Janssen, M. (2019). A Systematic Literature Study to Unravel Transparency Enabled by Open Government Data: The Window Theory. Public Performance \& Management Review, 43(3), 1-32. https://doi.org/10.1080/15309576.2019.1691025 
Moher, D., Liberati, A., Tetzlaff, J., Altman, D. G., Altman, D., Antes, G., Atkins, D., Barbour, V., Barrowman, N., Berlin, J. A., Clark, J., Clarke, M., Cook, D., D’Amico, R., Deeks, J. J., Devereaux, P. J., Dickersin, K., Egger, M., Ernst, E., ... Tugwell, P. (2009). Preferred reporting items for systematic reviews and meta-analyses: The PRISMA statement. PLoS Medicine, 6(7). https://doi.org/10.1371/journal.pmed.1000097

Moon, M. (2020). Shifting from Old Open Government to New Open Government: Four Critical Dimensions and Case Illustrations. Public Performance \& Management Review, 43(3), 535-559. https://doi.org/10.1080/15309576.2019.1691024

Mueller, B. (2020). Why public policies fail: Policymaking under complexity. EconomiA, 21(2), 311-323. https://doi.org/10.1016/j.econ.2019.11.002

Presidencia del Consejo de Ministros (2002). Ley N²7806 - Ley de Transparencia y Acceso a la Información Pública. https://www.peru.gob.pe/normas/docs/ley_27806.pdf.

Presidencia del Consejo de Ministros (2010). Decreto Supremo que aprueba la implementación del Portal de Transparencia Estándar en las Entidades de la Administración Pública DECRETO SUPREMO N ${ }^{\circ}$ 063-2010PCM. https://cdn.www.gob.pe/uploads/document/file/357116/DS_N_063-2010-PCM.pdf.

Presidencia del Consejo de Ministros (2017a). Modelo y Estratégia de datos abiertos gubernamentales. Cuatro Ases Línea Gráfica SAC. https://sgp.pcm.gob.pe/wp-content/uploads/2017/05/Modelo-y-Estrategia-DAG-del-Peru.pdf.

Presidencia del Consejo de Ministros (2017b). RM_N_035-2017-PCM Lineamientos para la implementación del Portal de transparencia estándar en las entidades de la Administración Pública. https://cdn.www.gob.pe/uploads/document/file/12705/RM_N_035-2017-PCM.pdf.

Presidencia del Consejo de Ministros (2018). Gobierno Abierto. https://sgp.pcm.gob.pe/wpcontent/uploads/2015/01/Fasciculo-4-Gobierno-Abierto.pdf.

Purwanto, A., Zuiderwijk, A.y Janssen, M. (2020). Citizen Engagement With Open Government Data. International Journal of Electronic Government Research, 16(3), 1-25. https://doi.org/10.4018/IJEGR.2020070101

Ruvalcaba-Gomez, E. y Renteria, C. (2020). Contrasting perceptions about transparency, citizen participation, and open government between civil society organization and government. Information Polity, 25(3), 323-337. https://doi.org/10.3233/IP-190185

Safarov, I. (2020). Institutional Dimensions of Open Government Data Implementation: Evidence from Transition Countries. Public Performance \& Management Review, 43(6), 1359-1389. https://doi.org/10.1080/15309576.2020.1805336

Sandoval-Almazan, R. (2019). Gobierno Abierto y Transparencia en México: estudio longitudinal 2015-2018. Innovar, 29(74), 115-131. https://doi.org/10.15446/innovar.v29n74.82097

Santos, L. y Santos, C. (2017). A study on the impact of non-operational mechanisms on the effectiveness of public information technology governance. Revista de Administração, 52(3), 256-267. https://doi.org/10.1016/j.rausp.2017.05.005

Schnell, S. (2020). Vision, Voice, and Technology: Is There a Global "Open Government" Trend? Administration \& Society, 52(10), 1593-1620. https://doi.org/10.1177/0095399720918316

Veres, C. (2020). Conceptual Model for Introducing Lean Management Instruments. Procedia Manufacturing, 46, 233237. https://doi.org/10.1016/j.promfg.2020.03.034

Widjajarto, A., Lubis, M. y Yunan, U. (2019). Architecture Model of Information Technology Infrastructure based on Service Quality at Government Institution. Procedia Computer Science, 161, 841-850. https://doi.org/10.1016/j.procs.2019.11.191

Zuiderwijk, A., Shinde, R. y Janssen, M. (2019). Investigating the attainment of open government data objectives: is there a mismatch between objectives and results? International Review of Administrative Sciences, 85(4), 645672. https://doi.org/10.1177/0020852317739115 